\title{
Educating Patients About Opioid Disposal: A Key Role for Perianesthesia Nurses
}

\author{
Katreena C. Merrill \\ Brigham Young University - Provo \\ Valynn Christina Haslam \\ Karlen E. (Beth) Luthy \\ Brigham Young University - Provo \\ Craig Nuttall \\ Brigham Young University - Provo, craig-nuttall@byu.edu
}

Follow this and additional works at: https://scholarsarchive.byu.edu/facpub

Part of the Other Nursing Commons

\section{Original Publication Citation}

Merrill, K. C., Haslam, V. C., Luthy, K. E. B., \& Nuttall, C. (2019). Educating patients about opioid disposal: A key role for perianesthesia nurses. Journal of PeriAnesthesia Nursing.

\section{BYU ScholarsArchive Citation}

Merrill, Katreena C.; Haslam, Valynn Christina; Luthy, Karlen E. (Beth); and Nuttall, Craig, "Educating Patients About Opioid Disposal: A Key Role for Perianesthesia Nurses" (2019). Faculty Publications. 5085. https://scholarsarchive.byu.edu/facpub/5085 


\title{
Educating Patients About Opioid Disposal: A Key Role for Perianesthesia Nurses
}

\author{
Katreena C. Merrill, PbD, RN, Valynn Christina Haslam, MS, FNP-C, \\ Karlen E. (Betb) Lutby, DNP, FNP-C, FAAN, FAANP, Craig Nuttall, DNP, FNP-C
}

\begin{abstract}
Purpose: To examine opioid prescription practices, patient use of opioids after laparoscopic cholecystectomy, and patient knowledge about disposal of unused medication.

Design: Mixed methods design with a chart review and structured phone interview.

Methods: Thirty-four patients were interviewed by phone and asked how many opioids were prescribed, bow many excess pills they had 6 to 10 days postoperatively, and if they knew how to dispose of them.

Findings: Average number of excess pills ranged from 0 to 42 (mean =14; $S D=11.7)$. Nearly half of patients $(47 \%)$ perceived the prescribed quantity as "too many." Seventy-one percent of patients using opioids for 5 days or less and $88 \%$ did not recall any instruction about medication disposal. Conclusions: Patients often have excess prescribed opioids after laparoscopic cholecystectomy, and they do not understand proper disposal of unused medications. Education about proper disposal of unused opioids medications is a key role of perianesthesia.
\end{abstract}

Keywords: laparoscopic cholecystectomy, opioid, medication disposal, prescription, education.

(C) 2019 by American Society of PeriAnesthesia Nurses

THROUGHOUT THE 1990S, a transformation of beliefs and attitudes surrounding pain management swept across the United States. Position statements from quality improvement agencies and others demanded health care professionals be more aggressive in treating pain and relieving suffering. In 1998, the Veteran's Health Adminis-

Katreena C. Merrill, PbD, RN, College of Nursing, Brigham Young University, Provo, UT; Valynn Christina Haslam, MS, FNP-C, Revere Health, Orem, UT; Karlen E. (Beth) Luthy, DNP, FNP-C, FAAN, FAANP, College of Nursing, Brigham Young University, Provo, UT; and Craig Nuttall, DNP, FNP-C, College of Nursing, Brigham Young University, Provo, UT.

Funding: This study was funded in part by a student scholarship provided to Ms. Haslam by Intermountain Healthcare. Conflicts of interest: none to report.

Address correspondence to Katreena C. Merrill, College of Nursing, Brigham Young University, 500 D Kimball Tower, Provo,UT 84602; e-mail address: katreena.merrill@byu.eddu. (C) 2019 by American Society of PeriAnesthesia Nurses $1089-9472 / \$ 36.00$

bttps://doi.org/10.1016/j.jopan.2018.12.008 tration implemented a process where pain was assessed as the "fifth vital sign," as recommended by the American Pain Society (APS), and severity was scored on a 0 to 10 scale. ${ }^{1}$ By 2001 , the Joint Commission on Accreditation of Healthcare Organizations mandated pain assessment and management standards as part of accreditation requirements. $^{2}$ Simultaneously, physicians in training were encouraged to prescribe opioids with little education about the patient safety consequences. ${ }^{3}$ As a result, prescriptions for opioid medications increased dramatically from 1991 to 2013. The sharp increase in number of prescribed opioids was paralleled by a dramatic increase in the number of opioid-related deaths. It is estimated that more than 200 million opioid prescriptions are filled annually in the United States, 2.5 million people misuse, and 16,500 to 19,500 deaths are related to unintentional opioid overdose. ${ }^{4}$

One common reason for prescribing opioids is to treat postoperative pain. Historically, surgical 
intervention was considered an invasive treatment option, resulting in significant pain, extended recovery time, and residual scarring. Development of laparoscopes and other sophisticated surgical devices, however, have provided means for lessinvasive surgical techniques. Gallbladder removal, one of the most frequently performed surgeries in the United States and once considered a major surgery, required a very large incision to open the abdomen and days spent in the hospital postoperatively. The laparoscopic approach to gallbladder removal (laparoscopic cholecystectomy) has now become the gold standard as it results in significantly shorter recovery time, minimal scarring, and less postoperative pain. ${ }^{5}$ Most patients who undergo laparoscopic cholecystectomy are discharged home on the same day.

Although the severity of pain and need for adequate treatment should be recognized, it is well documented that opioids are often overprescribed after invasive procedures. In 2011, researchers examining postoperative opioid use after urological surgery found $67 \%$ of patients were prescribed an excessive number of opioid pills postoperatively. ${ }^{6}$ In a more recently published study examining opioid use after outpatient orthopaedic surgery, health care providers prescribed up to $75 \%$ more opioids than the patient used. ${ }^{7}$ This type of overprescribing of opioids can result in misuse or abuse of unused medications. ${ }^{8}$

Multimodal approaches to pain management are often reported to manage pain, thus resulting in a decreased need for opioids. The American Society of Anesthesiologists (ASA) strongly supports multimodal pain management therapy including use of acetaminophen, nonsteroidal anti-inflammatory medications (NSAIDs), and calcium channel alpha 2 antagonists, such as gabapentin or pregabalin to augment opioid effectiveness. ${ }^{9}$ However, despite these recommendations, opioids remain the medication of choice for many prescribers. ${ }^{10}$

Opioids are effective analgesics, not by removing the pain stimulus or potentiating tissue healing, but by decreasing the central nervous system's perception of pain stimuli, thus increasing a patient's tolerance for pain. ${ }^{11}$ The euphoria associated with opioid use is a primary reason why opioids are highly addictive and, as a result, have high potential for misuse and abuse. Jones et $\mathrm{al}^{12}$ found those who abused opioids on a short-term basis identified their supply sources as family and friends who had leftover medication. Thus, teaching the public about proper disposal of unused opioids becomes extremely important in addressing the opioids crisis. ${ }^{8}$ Both Bates et $\mathrm{al}^{6}$ and Kumar et $\mathrm{al}^{7}$ found education regarding proper disposal of leftover opioid prescription medication was lacking.

Several states of United States help fund campaigns to educate consumers on proper use and disposal of opioids, including Georgia's "Generation Rx Project," Ohio's "Prescription for prevention," Wisconsin's "Good Drugs Gone Bad," and Utah's "Use Only As Directed." These media campaigns aim to educate and encourage the public to help fight the misuse of opioids by appropriately using and then disposing of medications. ${ }^{13}$

One barrier to effective education is the inconsistency between state laws and regulations regarding appropriate disposal of opioids. ${ }^{14}$ The Food and Drug Administration recommends using Drug Enforcement Agency (DEA) sponsored TakeBack programs and facilities as the ideal method of opioid medication disposal. ${ }^{15}$ Nevertheless, TakeBack programs and safe drop boxes for unused medication were not widely available until 2014 , when the DEA expanded authorization for takeback facilities to include hospitals and pharmacies. ${ }^{16}$ Because the DEA only recently expanded programs, health care providers, including physicians, nurses, and pharmacists may not be knowledgeable of what disposal facilities are now available in their own communities.

\section{Purpose Statement}

The purpose of this descriptive research study was to examine providers' opioid prescriptive practices, and patients' pain levels and knowledge about appropriate disposal of unused opioids after uncomplicated laparoscopic cholecystectomy.

\section{Research Questions}

1. How many opioid pills do prescribers order after an uncomplicated laparoscopic cholecystectomy? 
2. How do patients describe their pain level and pain management 6 to 10 days after uncomplicated laparoscopic cholecystectomy?

3. What are the patient perceptions of the amount of prescribed pain medication after uncomplicated laparoscopic cholecystectomy?

4. What is the patients' reported knowledge of how to dispose of unused opioids?

\section{Methods}

\section{Sample and Setting}

Researchers recruited a convenience sample of 50 patients scheduled for elective laparoscopic cholecystectomy to participate in this descriptive study. Surgeries were performed in a same day surgery department at a tertiary care hospital in Utah between November 2016 and February 2017. Patients were included if they were scheduled for a nonemergent, uncomplicated laparoscopic cholecystectomy who spoke English, were aged 18 to 65 years, and were determined by their physician to be competent. Patients were excluded if they were currently being treated for chronic pain, unplanned in-patient admission postoperatively, self-disclosed history of opioid or illicit drug abuse, and a surgical procedure complicated by biliary duct obstruction requiring endoscopic retrograde cholangiopancreatography.

During preoperative check-in and before any medication delivery, two designated preoperative nurses approached the patient for consent. Patients signed a written consent after the study was explained and questions answered. During the consent process, patients' provided a phone number and agreed to be contacted approximately 6 to 10 days postoperatively.

Before the postoperative phone call, the operative report was reviewed to determine if the patient met inclusion criteria. Patients' demographic information (age, ethnicity, gender, and date of surgery) was extracted from the electronic health record and additional information was gathered using a structured interview by phone, 6 to 10 days postoperatively. The researcher attempted to contact participants three times before being eliminated from the study.

\section{Description of Procedures for Protection of Participants' Rights}

Institutional review board approval via expedited review was received from the hospital before the study. The institutional review board determined the study to be minimal risk. Two research assistants, trained in research ethics and compliance who worked in the same day surgery department of the hospital assisted with the initial patient consent. Patients were provided a written consent form and given an opportunity to ask questions before their scheduled laparoscopic cholecystectomy. Those who decided to participate, signed their name on the consent, agreed to have the principle investigator review their chart, and receive a phone call 6 to 10 days after surgery. The completed consent forms were kept in a locked filing cabinet in the same day surgery department. The principle investigator collected the consents, reviewed the patients' electronic record, and called the patients at home. A second verbal consent was read to the patient before the telephone interview. Hard copy data were kept in a locked filing cabinet and electronic data were kept in a password-protected file.

\section{Instrument}

There are no validated instruments measuring opioid prescription practices after same day surgery. The instrument consisted of a structured questionnaire developed by the researchers based on literature review and expert opinion. The questionnaire included 11 questions plus demographic information (age, gender, ethnicity, date of surgery, and surgeon). Questions included self-rated pain levels with and without activity ( 0 to 10 Likert scale), the number of prescribed opioid pills after surgery, the number of opioid pills taken up until the time of the interview, other medications used for pain control, side effects experienced, a rating of the amount of opioids prescribed (too few, just right, or too many), and whether the patient had received instructions about disposal of unused opioids.

\section{Data Analysis}

The principle investigator transcribed the data and entered into a password-protected Excel spreadsheet. The data were then entered into SPSS version 24 for analysis by the statistician who 
completed a descriptive analysis for quantitative variables (mean, standard deviation, range, and distribution of the data). ${ }^{17}$ Because of the small sample size and the fact that the data were not normally distributed, a Spearman $\rho$ correlation was used to assess the relationship among age, pain, and the number of opioid pills used.

\section{Results}

Fifty patients were approached to participate in the study. Forty-two participants were enrolled in the study preoperatively ( $84 \%$ response rate). Three patients were excluded because of complications after surgery and five patients did not answer follow-up phone calls. The final sample included 34 patients (final response rate $68 \%$ ). Participants were mostly White (97\%), female (91\%), and an average age of 36.8 years (range 20 to 65 years). Ten different health care providers prescribed opioids for study participants postoperatively.

\section{Pain Level and Pain Management}

Patients reported their average pain on a 10-point Likert scale with and without activity at the time of the follow-up phone call. With no activity, patients' mean reported pain was $2.0(\mathrm{SD}=1.8)$. With activity, patients reported average pain score was 4.1 $(S D=2.3)$. Patients reported using both prescribed opioid and over the counter analgesics to manage their postoperative pain. Fifty-eight $(8 \% ; n=20)$ patients used a multimodal approach with ibuprofen or acetaminophen taken in conjunction with opioids. The average number of days of opioid use after surgery was 4.2 with $71 \%(n=29)$ of patients using opioids for 5 days or less. At the time of the follow-up phone call, patients reported having taken a total of about 11 opioids to control their pain (mean $[\mathrm{M}]=10.9$; $\mathrm{SD}=8.2$; range 0 to 26). In addition, about $8 \%(n=3)$ of the patients in this study reported using no opioids after the day of surgery.

\section{Opioids Prescribed After Surgery}

From the patients' account, health care providers prescribed the subjects in this study between 12 and 50 opioid pills after their uncomplicated laparoscopic cholecystectomy $(\mathrm{M}=27$; $\mathrm{SD}=8)$. At the time of the follow-up phone call, patients reported having between 0 and 42 leftover pills $(\mathrm{M}=14 ; \mathrm{SD}=11.7)$.
Patients were asked if they felt they were prescribed too few, just right, or too many opioid pills after surgery. Forty-seven percent of patients $(\mathrm{n}=16)$ felt they were prescribed "too many," whereas only $11.8 \%(\mathrm{n}=4)$ indicated they were prescribed "too few" opioids with $41 \%(\mathrm{n}=14)$ indicating the amount of opioids prescribed was "just right." Of the participants who rated the prescribed quantity as just right, 11 participants had anywhere from 2 to 19 opioid leftover pills $(M=8.1)$. For the 16 patients who reported the prescribed quantity as too many, they reported having 0 to 42 opioid leftover pills $(M=21.8)$. There was no relationship between age and the number of days needing opioids $(r=0.025 ; P=.8)$ and pain level with or without activity $(r=-0.06$ to $0.027 ; P=.7$ to .8 ). There was a moderate positive correlation with age and the number of leftover pills $(r=0.387 ; P=.026)$.

Most patients $(64 \% ; n=22)$ reported side effects (nausea, itchy, sleepy, dizzy, and constipation) resulting from opioid administration and half of all participants agreed that medication side effects influenced the number of opioid pills they used to treat their pain.

\section{Knowledge of Disposal}

Eighty-eight percent $(n=30)$ reported that they were not informed about disposal of unused opioids. A few $(n=2)$ reported not being instructed personally by either a health care provider or nursing staff, but instead referred to statesponsored programs focused on medication safety, stating, "I've seen the billboards." A small number of participants volunteered what they thought was the proper way to dispose of surplus supply, stating it was appropriate to "flush them" $(n=5)$, "take them somewhere" $(n=1)$, and "use a drop box/take-back facility" $(n=6)$. Others $(n=20)$ in the study indicated they had no knowledge of how to appropriately dispose of unused opioid pills. One participant indicated plans to keep the remaining opioids in the home, stating, "I feel safer with a few leftover."

\section{Discussion}

In this study, we identified three key findings: Some providers continue to use opioids as the 
primary treatment for pain management postoperatively instead of recommending patients use a multimodal approach, the quantity of opioid pills prescribed is often more than necessary, and patients are not routinely provided with adequate education on safe disposal of unused medications. These findings are important because the same behaviors, which have been shown to contribute to opioid abuse, continue in spite of recommendations and guidelines commissioned by the APS, the American Academy of Pain Medicine, and the American Medical Association. ${ }^{18-20}$

It is important to note that some patients (such as those with chronic pain) may need opioid approaches to postoperative pain management. However, for most patients, a multimodal approach for analgesia is recommended for many different types of pain, including postoperative pain. ${ }^{21}$ The ASA encourages an around-the-clock regimen of NSAID, cyclooxygenase-2 inhibitors (COXIB), or acetaminophen as primary coverage for pain management, with opioids used as adjunct therapy for breakthrough pain.' In fact, solely nonopioid approaches have been shown to provide comparable pain relief when used in some postoperative situations, such as after tonsillectomy in children and after carpal tunnel release in adults. ${ }^{22,23}$ Similarly, some of our study participants (11.8\%) found adequate pain relief using only NSAIDs or acetaminophen for pain control after surgery, without any opioids. Despite multimodal therapy recommendations by numerous pain management experts (ie, ASA, APS, and so forth), the primary method of treatment for pain after laparoscopic cholecystectomy in our study was opioids.

Many of our study participants indicated being prescribed too many opioid pills after laparoscopic cholecystectomy. Our data are supported by a much larger study conducted by Hill et $\mathrm{al}^{24}$ who found wide variation and overprescribing of opioids after common surgeries. Former surgeon General Vivek Murthy suggested one reason why physicians may still overprescribe opioids: he recalls being in medical school nearly two decades ago, when medical students were taught to aggressively treat pain with opioids-without adequate training, support, or accurate information about the addictive potential of opioids. ${ }^{3}$ Furthermore, Stanek et $\mathrm{al}^{25}$ suggested nonspecific, generalized opioid prescription recommendations currently available are insufficient to provide meaningful counsel for prescribers. Our study findings suggest prescribing 19 opioid pills after laparoscopic cholecystectomy was adequate for $81 \%$ ofourparticipants. Similarly, Hilletal ${ }^{24}$ recommended prescribing only 15 opioid pills after laparoscopic cholecystectomy, which would satisfy the opioid demand of $80 \%$ of postoperative patients in their study.

Overprescribing opioids for postoperative pain management results in an excess of unused medication. This practice leaves patients with the decision to either dispose or store the remaining opioid medication. In this study, $88 \%$ of our participants indicated that they did not recall receiving any information about how to dispose of unused medication appropriately, and although we did not specifically ask what they intended to do with excess opioid pills, many patients stated they would be storing the medication for future use. This is consistent with findings from Bates et $\mathrm{al}^{6}$ who determined $92 \%$ of their study participants recalled no disposal instructions and $90 \%$ of their study participants indicated they planned to keep excess opioid pills.

\section{Limitations}

An a priori power analysis indicated 100 subjects would be needed to reach statistical significance. Therefore, the limitations of this study include an insufficiently small sample size and a homogeneous sample from one hospital. Most of the study participants were Caucasian females. In addition, the type and concentration of prescribed opioid medication were not collected (ie, oxycodone $5 \mathrm{mg}$ vs hydrocodone $7.5 \mathrm{mg}$ ). Another limitation of our study was that we only included healthy patients. This lack of adjustment for participant comorbidities such as anxiety, chronic pain, depression, or other health concerns that have been shown to influence the perception of pain and account for the need for fewer opioids needed postoperatively. $^{26}$

\section{Recommendations for Perianestbesia Nurses}

There are three main recommendations for perianesthesia nurses that resulted from this study: encouraging multimodal pain management, updating postoperative protocols, and teaching about proper disposal. 
Using a variety of medications to manage postoperative pain may minimize the number of opioids needed to relieve pain. Congruent with the ASA guidelines, nurses should implement evidencebased best practice protocols and educate their patients about around-the-clock regimens of NSAIDs (ie, ibuprofen) and acetaminophen as the primary analgesics after laparoscopic cholecystectomy (if not contraindicated). A multimodal pain management regimen may help decrease the need for opioids while still achieving adequate analgesia for patients after laparoscopic cholecystectomy. $^{27}$ Perianesthesia nurses should review current patient education materials to ensure that the latest evidence-based information including multimodal pain management is included. In addition, it is important for perianesthesia nurses to remember that some patients, such as those with chronic pain or other comorbidities, may need opioids for postoperative pain management.

Many ambulatory surgery settings use standardized protocols after surgery. Some researchers are trying to determine the appropriate opioid regimen for use after each type of surgery. For example, Kim et $\mathrm{al}^{28}$ provided a general guideline for postoperative opioid prescription quantity based on surgical type and anatomic location. Stanek et $\mathrm{al}^{25}$ suggested a simple educational-assist device, in addition to changing postoperative order sets, may be an alternative pathway in affecting overprescribing habits. Others examined genetic profiles, which resulted in individualized pain management plans. ${ }^{27}$ Perianesthesia nurses are key members of the interdisciplinary team and can recommend revision to order sets that include opioid medications. Furthermore, these protocols should include patient education about proper disposal.

Unscripted responses from our study participants suggest that opioid-related media campaigns are somewhat effective in educating about proper opioid medication disposal. However, more direct and personalized instruction from health care providers was lacking in our study. Nurses need to know where take-back facilities are located in the community they serve so that patients can be accurately educated. Education about proper disposal of leftover opioids needs to become a routine part of prescribing and administering any opioid medication. Perianesthesia nurses need to take the initiative to update current patient education materials to include proper disposal of unused opioids.

\section{Recommendations for Future Research}

Although there are many assumptions and opinions about why health care providers continue to overprescribe opioid medication, additional research is needed to understand the rationale and the barriers influencing overprescribing practices. More research is needed about which types of patients (such as those with chronic pain) should be prescribed opioids and those who would benefit by multimodal approaches to postoperative pain management. It would also be useful to investigate the knowledge and attitudes of health care providers about proper medication disposal. Finally, as medication disposal programs and state-sponsored campaigns continue to expand, additional research is needed to analyze the effectiveness of the Take-Back program and public education.

\section{Conclusions}

Pain management is an ethical responsibility for health care providers. We investigated the prescribing pattern of opioids after uncomplicated laparoscopic cholecystectomy in patients without comorbidities. We identified that patients did not use multimodal approaches for postoperative pain management, were overprescribed opioids, and did not know proper disposal methods for unused opioids. Information from this study should be used to change prescribing practices and inform future research.

\section{References}

1. United States Department of Veterans Affairs. Geriatrics and Extended Care Strategic Healthcare Group, National Pain Management Coordinating Committee. In: Pain as the 5th Vital Sign Toolkit, Rev. ed.. Available at: www.va.gov/painmanagement/docs/ pain_as_the_5th_vital_sign_toolkit.pdf. Accessed June 4, 2018.
2. Joint Commission on Accreditation of Healthcare Organizations. Joint Commission on Accreditation of Healthcare Organizations Pain Standards for 2001. Available at: https:// www.jointcommission.org/assets/1/6/2001_Pain_Standards. pdf. Accessed June 4, 2018. 
3. Murthy V. The Surgeon General's Call to End the Opioid Crisis. Available at: www.turnthetiderx.org. Accessed June 4, 2018.

4. National Institute of Health, National Institute on Drug Abuse, Senate Caucus on International Narcotics Control. America's Addiction to Opioids: Heroin and Prescription Drug Abuse. Available at: https://www. drugcaucus.senate.gov/content/senate-caucus-internationalnarcotics-control-hearing-america $\% \mathrm{E} 2 \% 80 \% 99$ s-addiction-opi oids-heroin-and. Accessed June 4, 2018.

5. Soper NJ, Malladi P. Laparoscopic cholecystectomy. In: Ashley SW, Chen W, eds. UpToDate. Waltham, Mass: UpToDate. Available at:www.uptodate.com. Accessed June 4, 2018.

6. Bates C, Laciak R, Southwick A, Bishoff J. Over prescription of postoperative narcotics: A look at postoperative pain medication delivery, consumption and disposal in urological practice. J Urol. 2011;185:551-555.

7. Kumar K, Gulotta LV, Dines JS, et al. Unused opioid pills after outpatient shoulder surgeries given current perioperative prescribing habits. Am J Sports Med. 2017;45:636-641.

8. United States Department of Health and Human Services, Centers of Disease Control and Prevention. Adult use of prescription opioid pain medications-Utah, 2008. MMWR. 2010;59:153-157.

9. American Society of Anesthesiologists. Practice guidelines for acute pain management in the perioperative setting: An updated report by the American Society of Anesthesiologists task force on acute pain management. Anesthesiology. 2012;116: 248-273.

10. Garimella V, Cellini C. Postoperative pain control. Clin Colon Rectal Surg. 2013;26:191-196.

11. National Institute of Health, National Institute on Drug Abuse. Which Classes of Prescription Drugs are Commonly Misused: Opioids? Available at: https://www.drugabuse.gov/ publications/research-reports/misuse-prescription-drugs/whichclasses-prescription-drugs-are-commonly-misused. Accessed June 4, 2018.

12. Jones CM, Paulozzi LJ, Mack KA. Sources of prescription opioid pain relievers by frequency of past-year non-medical use: United States, 2008-2011. JAMA Intern Med. 2014;174:802-803.

13. Substance Abuse and Mental Health Services Administration. Substance Misuse Prevention Media Campaigns. Available at:https://www.samhsa.gov/capt/tools-learning-resources/ prevention-media-campaigns. Accessed June 4, 2018.

14. Food and Drug Administration. Medicine Disposal: Questions and Answers. Available at:https://www.fda.gov/Drugs/ ResourcesForYou/Consumers/BuyingUsingMedicineSafely/Ens uringSafeUseofMedicine/SafeDisposalofMedicines/ucm 186188 .htm, . Accessed June 4, 2018.

15. United States Department of Justice, Office of Public Affairs. Attorney General Holder Announces New Drug
Take-back Effort to Help Tackle Rising Threat of Prescription Drug Addiction and Opioid Abuse. Available at:https://www. justice.gov/opa/pr/attorney-general-holder-announces-new-drugtake-back-effort-help-tackle-rising-threat, . Accessed June 4, 2018.

16. Herring ME, Shah SK, Shah SK, Gupta AK. Current regulation and modest proposals regarding disposal of unused opioids and other controlled substances. J Am Osteopath Assoc 2008; 108:338-343.

17. IBM Corp.. IBM SPSS Statistics for Windows, Version 24. O. Armonk, NY: IBM Corp; 2016.

18. Chou R, Fanciullo GJ, Fine PG, et al. Clinical guidelines for the use of chronic opioid therapy in chronic noncancer pain. J Pain. 2009;10:113-130.

19. Hooten M, Thorson D, Bianco J, et al. Pain: Assessment, Non-opioid Treatment Approaches and Opioid Management. Bloomington (MN): Institute for Clinical systems Improvement (ICSI):160. Available at:https://www.guideline.gov/summaries/ summary/50568, . Accessed June 4, 2018.

20. American Medical Association. List of State \& Medical Society Opioid Education Resources. Available at:https:// www.ama-assn.org/content/state-medical-society-opioid-educa tion-resources, . Accessed June 4, 2018.

21. National Pharmaceutical Council. Pain: Current Understanding of Assessment, Management, and Treatments. Available at: http://www.npcnow.org/system/files/research/ download/Pain-Current-Understanding-of-Assessment-Manage ment-and-Treatments.pdf. Accessed June 4, 2018.

22. Baugh RF, Archer SM, Mitchell RB, et al. Clinical practice guideline: Tonsillectomy in children. Otolaryngol Head Neck Surg. 2010;44:S1-S30.

23. Chapman T, Kim N, Maltenfort M, Ilyas AM. Prospective evaluation of opioid consumption following carpal tunnel release surgery. Hand (NY). 2017;12:39-42.

24. Hill MV, McMahon ML, Stucke RS, Barth RJ. Wide variation and excessive dosage of opioid prescriptions for common general surgical procedures. Ann Surg. 2017;265:709-714.

25. Stanek J, Renslow M, Kalliainen L. The effect of an educational program on opioid prescription patterns in hand surgery: A quality improvement program. J Hand Surg Am. 2015;40: 341-346.

26. Craven $\mathrm{P}$, Cinar O, Madsen T. Patient anxiety may influence the efficacy of ED pain management. Am J Emerg Med. 2012;31:313-318.

27. Manworren RCB. Multimodal pain management and the future of a personalized medicine approach to pain. $A O R N J$. 2015;101:307-318.

28. Kim N, Matzon JL, Abboudi J, et al. A prospective evaluation of opioid utilization after upper-extremity surgical procedures: Identifying consumption patterns and determining prescribing guidelines. J Bone Joint Surg Am. 2016;98:e89. 